\title{
Bilateral Choroidal Metastasis as the Initial Clinical Presentation of Breast Carcinoma: A Case Report
}

\author{
Maha N.M. Aljundi, MD \\ Department of Ophthalmology, King Abdul-Aziz University, Jeddah, Saudi Arabia
}

DOI: $10.36348 /$ sjm.2020.v05i11.004 $\quad$ | Received: 05.11.2020 | Accepted: 14.11.2020 | Published: 25.11.2020

*Corresponding Author: Maha N.M. Aljundi

\section{Abstract}

Background: We report a rare case in which metastatic choroidal tumor (presented as a total retinal detachment) in one eye and asymptomatic choroidal tumors of the other eye were found to be the initial clinical presentation of breast cancer. Clinical Presentation: We report a case in which a choroidal tumor presented as a total retinal detachment was found to be the initial clinical presentation of breast cancer. Conclusions: The importance of detailed history taking and careful assessment of patients presenting with ocular tumors, in order to accurately detect the primary tumor and decide on the proper treatment is highlighted in this case report.

Keywords: Choroidal metastasis, Retinal detachment, Breast cancer.

Copyright () 2020 The Author(s): This is an open-access article distributed under the terms of the Creative Commons Attribution 4.0 International License (CC BY-NC 4.0) which permits unrestricted use, distribution, and reproduction in any medium for non-commercial use provided the original author and source are credited.

\section{INTRODUCTION}

Breast cancer was found to be the primary tumor in $80-90 \%$ of female patients with intraocular metastasis [9]. The typical presentation of a breast cancer patient is usually due to an abnormal mammograph or a breast lump. In this case report we present a rare case in which, a total retinal detachment due to choroidal metastases in one eye and asymptomatic choroidal tumors in the other eye are the initial clinical presentation of breast cancer.

\section{CASE PRESENTATION}

A 50 year old female presented to the emergency department, complaining of a gradual loss of vision in the left eye over the past 14 days, a sudden complete loss of vision in the left eye on the day of the presentation and redness of the right eye. Her background medical history was otherwise unremarkable. On examination, visual acuity was 20/20 sc in the right eye, using the Snellen optotype, and defective light projection in the left eye. Other than redness of the right eye, the anterior segments in both eyes were unremarkable. Intraocular pressure was within normal limits in both eyes. On dilated fundoscopic examination, there was a total retinal detachment of the left eye; the severe elevation of the retina was yellowish-white in color and directly behind the lens (Figure 1a). With suspicion of a choroidal tumor, the patient was informed of the findings and the possible differential diagnoses and admitted to having hardening and ulceration in the left breast over the past year. Patient had a positive family history for breast cancer; she however did not seek medical help. A dilated fundus exam of the right eye revealed a retinal detachment and a choroidal tumor over the total temporal side of the retina (Figure $2 \mathrm{a}$ ), which was $4 \mathrm{~mm}$ away from the fovea, plateau-shaped, and yellowishwhite in color (Figure 2b), as well as a second choroidal tumor nasally to the optic nerve that was around 4 optic disc diameter in size (Figure 2c). B-scan sonography of the right eye showed a choroidal mass (Figure 2d).

The patient was referred to the obstetrics and gynecology (Ob-Gyn) breast unit. After a thorough physical examination, mammogram, and an ultrasound of both breast an ulcerated breast mass was found in the left breast as well as hard skin masses on both breasts and on the lower back.

The patient had multiple diagnostic procedures and investigations done (for eg. CT-scan and an MRI). She was diagnosed with stage IV Adenocarcinoma of both breasts with lymphatic, skin, pulmonary, thoracic and musculoskeletal metastases. She received palliative anti-hormonal therapy with Letrozo, as well as palliative Radiotherapy of the orbits over two weeks period.

In the one month follow up after the radiotherapy; the patient reported an improved vision on the left eye (Hand motion sc). The visual acuity of the right eye was stable $(20 / 20 \mathrm{sc})$. The fundus pictures showed improvement in the retinal detachment on the 
left eye (Figure 1b), as well as regression and reduction in the size of the tumors on the right eye (Figure 3a).

In the two months follow up after the radiotherapy, there was complete reduction of the tumors on both eyes (Figure 1c, d, e and Figure 3b, c) and the visual acuity was preserved in the right eye $20 / 20$ and improved on the left eye from defective light projection to $20 / 200$.

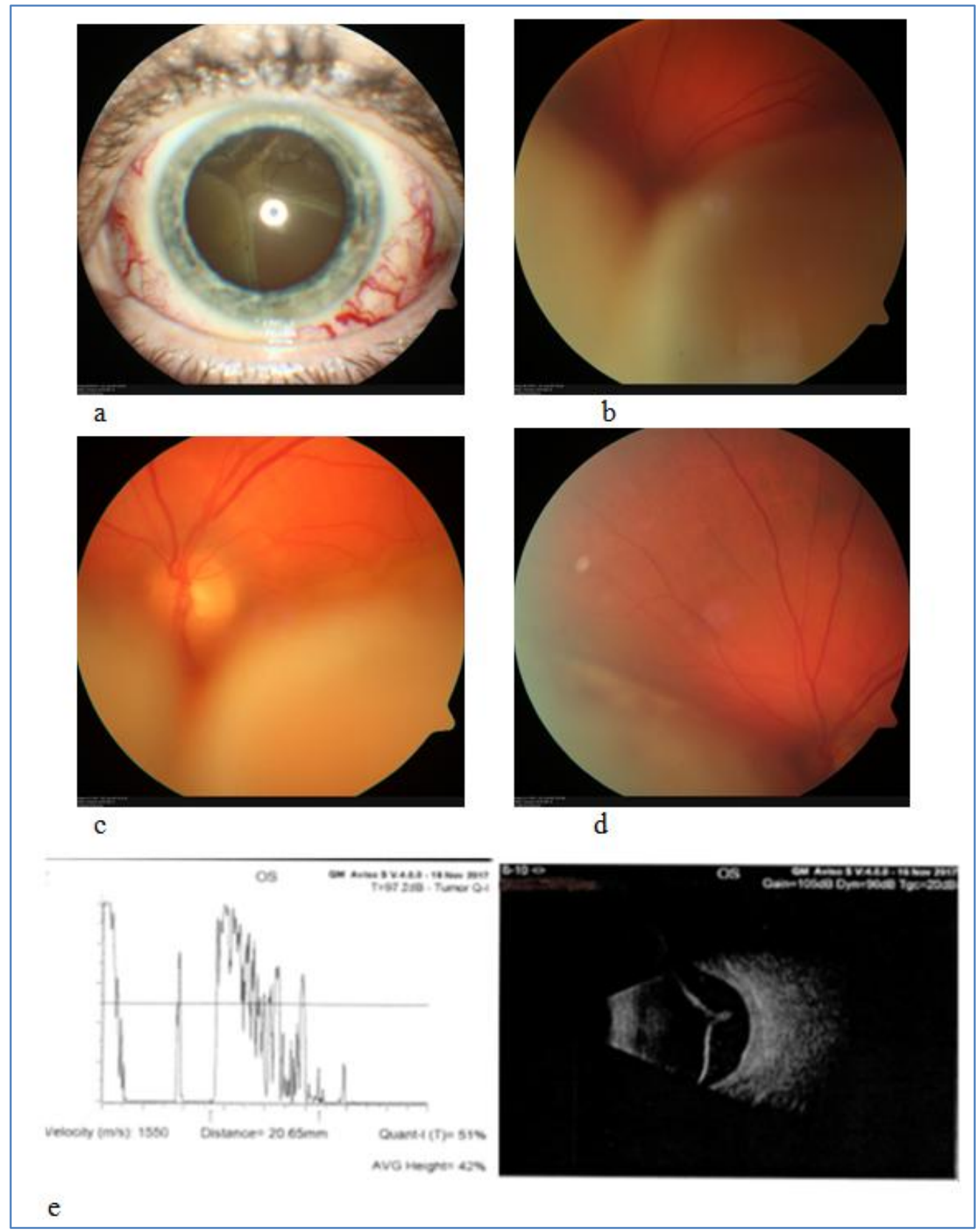

Figure 1: Left eye.

a. Total retinal detachment.

b. Regression of the choroidal tumor (1 month post radiotherapy).

c. Regression of the choroidal tumor ( 2 months post radiotherapy).

d. Regression of the choroidal tumor ( 2 months post radiotherapy).

e. A-scan and B-scan showing retinal detachment with regression of the choroidal tumor ( 2 months post radiotherapy). 

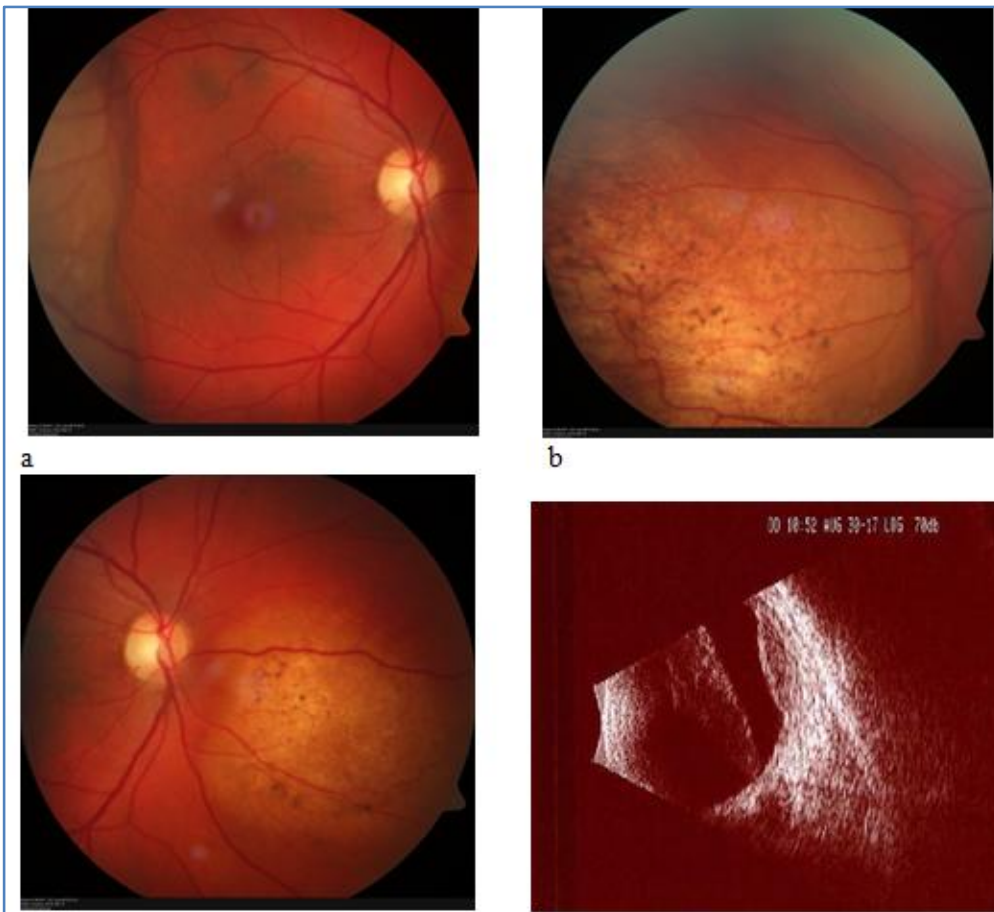

b

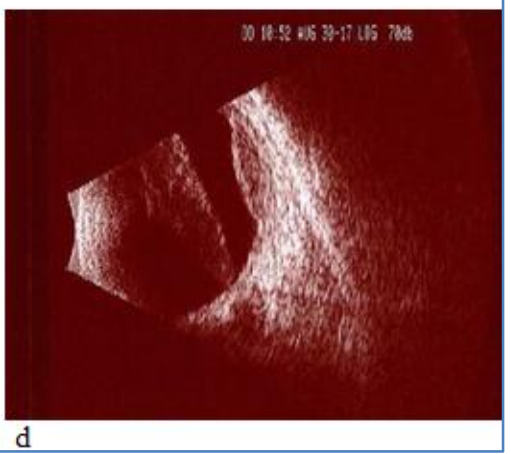

Fig-2: Right eye (at the time of the diagnosis)

a. Choroidal tumor $4 \mathrm{~mm}$ temporal to the fovea.

b. Yellowish grey, plateau- shaped choroidal tumor.

c. Choroidal tumor nasal to the optic nerve.

d. Choroidal Tumor on B-scan

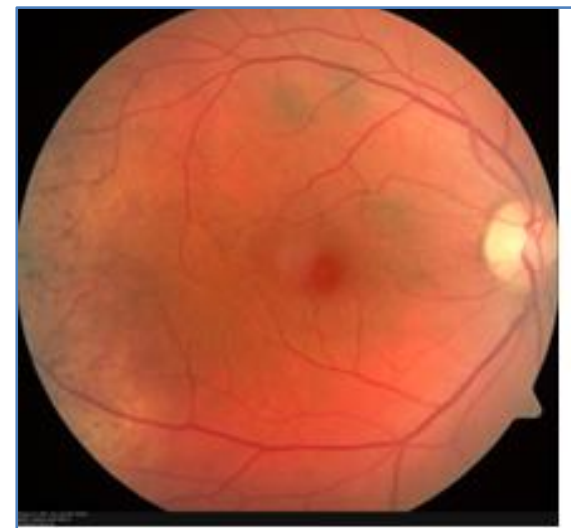

a

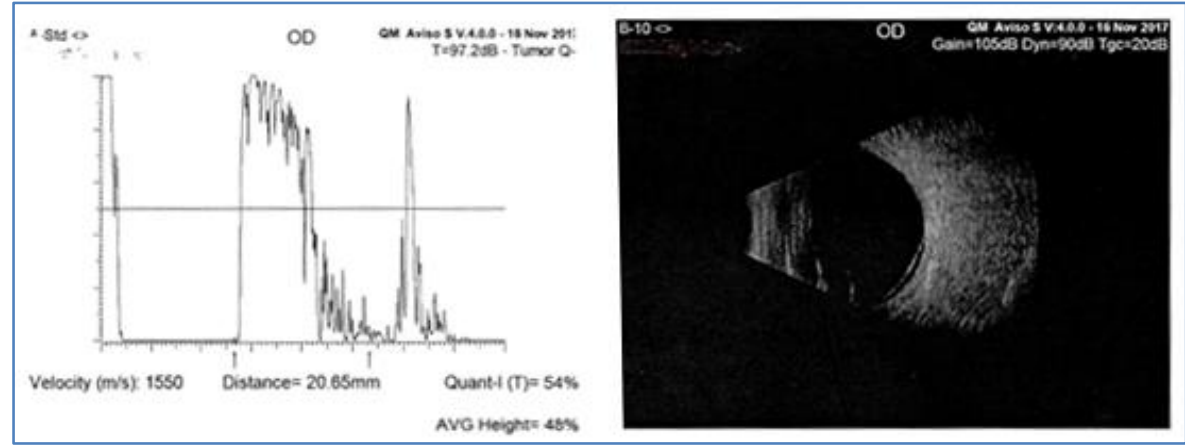

c

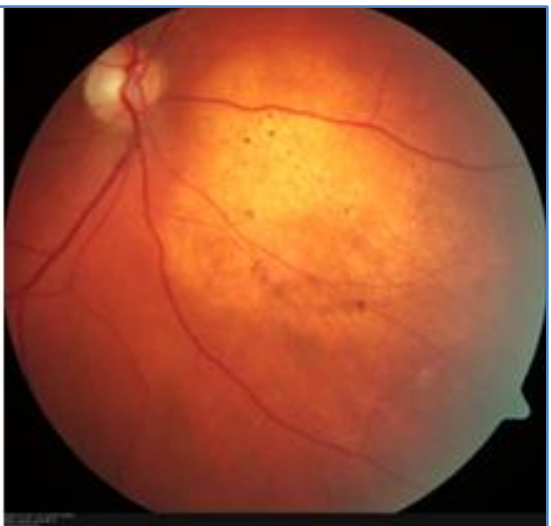

b

Fig-3: Right eye follow up

a. Regression of the choroidal tumor (1 month post radiotherapy).

b. Regression of the nasal choroidal tumor ( 2 months post radiotherapy).

c. A- scan and B- scan 2 months after radiotherapy 


\section{METHODS}

A 50 year old female presented with total retinal detachment due to a choroidal tumor on the left eye and multiple choroidal tumors on the right eye was clinically evaluated with visual acuity testing, slit lamp exam, fundus photography, B scan sonography. She was then referred to the breast unit in the Ob-Gyn department where she received a thorough physical exam, mammogram, breasts sonography, CT-scan and MRI (for breast cancer staging workup).

\section{DISCUSSION}

Breast cancer is the most common cancer that affects females in the world [4]. Most patients in countries with well-developed cancer screening programs present because of an abnormal mammogram [5]. A breast mass that is not detected on mammogram (mammographically occult disease) is the reason why $15 \%$ of women are diagnosed with breast cancer [5]. In addition, $30 \%$ present as interval cancers (with a breast mass noticed in the interval between mammorgams) [5].

The initial clinical presentation of the breast cancer in the patient in this case is a total retinal detachment as a result of the choroidal metastases, which is extremely rare [1-3]. The incidence of asymptomatic choroidal metastases as the single site of metastases in disseminated breast cancer is $5 \%$, and increases to $11 \%$ when there is more than one organ involved in the metastatic spread [6].

A thorough history taking and systemic examination of patients presenting with choroidal tumors is essential to differentiate metastases from possible choroidal melanoma and to detect the primary tumor. Breast cancer was found to be the primary tumor in $80-90 \%$ of female patients with intraocular metastasis $[9,10]$.

A proper diagnosis is important to determine the right therapy and to avoid unnecessary enucleation. Choroidal metastases in breast cancer was found to regress with aromatase inhibitors in patients with hormone receptor-positive breast cancer [7]. Lesion regression with EBRT was observed in $85-93 \%$ of patients [8]. Other treatment options include plaque brachytherapy, gamma knife radiosurgery, cyber knife radiotherapy, proton beam radiotherapy and intravitreal Bevacizumab (eg.11). The patient in this case report received orbital radiotherapy of 10 daily fractioned treatments for a total of $30 \mathrm{~Gy}$. The lesions in both eyes have regressed with this treatment, visual acuity was preserved in the right eye 20/20 and improved on the left eye from defective light projection to 20/200 two months after the radiation [11].

\section{CONCLUSION}

We present an atypical and rare first clinical manifestation of breast cancer. This case report highlights the importance of detailed history taking and careful assessment of patients presenting with ocular tumors, in order to accurately detect the primary tumor and decide on the proper treatment.

\section{REFERENCES}

1. Chen, Y. R., Lin, T. H., Chan, S. M., Chen, S. W., Yang, Y. A. N. G. S. E. N., Ho, K. C., \& Young, J. H. (1998). Bilateral choroidal metastases as the initial presentation of a small breast carcinoma: a case report. Zhonghua yi xue za zhi= Chinese medical journal; Free China ed, 61(2), 99-103.

2. Williams, N. J., Leris, A. C. A., Kouriefs, C., Mokbel, K. M., Hungerford, J., \& Carpenter, R. (2000). Choroidal metastasis - the initial presentation of breast carcinoma. European journal of surgical oncology, 26(8), 817-818.

3. O'Gorman, C. S., Awad, Z. T., \& Given, H. F. (2001). Bilateral choroidal metastases as the initial presentation of breast carcinoma. Irish Medical Journal, 94(3), 85-86.

4. Bray, F., Ferlay, J., Soerjomataram, I., Siegel, R. L., Torre, L. A., \& Jemal, A. (2018). Global cancer statistics 2018: GLOBOCAN estimates of incidence and mortality worldwide for 36 cancers in 185 countries. CA: a cancer journal for clinicians, 68(6), 394-424.

5. Esserman, L. J., Shieh, Y., Rutgers, E. J., Knauer, M., Retèl, V. P., Mook, S., ... \& van't Veer, L. J. (2011). Impact of mammographic screening on the detection of good and poor prognosis breast cancers. Breast cancer research and treatment, 130(3), 725-734.

6. Wiegel, T., Kreusel, K. M., Bornfeld, N., Bottke, D., Stange, M., Foerster, M. H., \& Hinkelbein, W. (1998). Frequency of asymptomatic choroidal metastasis in patients with disseminated breast cancer: results of a prospective screening programme. British Journal of Ophthalmology, 82(10), 1159-1161.

7. Manquez ME, Brown MM, Shields CL, Shields JA. Management of choroidal metastases from breast carcinomas using aromatase inhibitors. Current opinion in ophthalmology. 2006 Jun 1;17(3):251-6.

8. Stephens, R. F., \& Shields, J. A. (1979). Diagnosis and management of cancer metastatic to the uvea: a study of 70 cases. Ophthalmology, 86(7), 13361349.

9. Freedman, M. I., \& Folk, J. C. (1987). Metastatic tumors to the eye and orbit: patient survival and clinical characteristics. Archives of Ophthalmology, 105(9), 1215-1219.

10. Shields, C. L., Shields, J. A., Gross, N. E., Schwartz, G. P., \& Lally, S. E. (1997). Survey of 520 eyes with uveal metastases. Ophthalmology, 104(8), 1265-1276.

11. Chen, C. J., McCoy, A. N., Brahmer, J., \& Handa, J. T. (2011). Emerging treatments for choroidal metastases. Survey of ophthalmology, 56(6), 511521. 